\title{
Endothelial cells enhance the in vivo bone-forming ability of osteogenic cell sheets
}

\author{
Rogério P Pirraco ${ }^{1,2,3}$, Takanori Iwata', Toshiyuki Yoshida ${ }^{1}$, Alexandra P Marques ${ }^{2,3}$, Masayuki Yamato ${ }^{1}$, Rui L Reis ${ }^{2,3}$ \\ and Teruo Okano ${ }^{1}$
}

Addressing the problem of vascularization is of vital importance when engineering three-dimensional (3D) tissues. Endothelial cells are increasingly used in tissue-engineered constructs to obtain prevascularization and to enhance in vivo neovascularization. Rat bone marrow stromal cells were cultured in thermoresponsive dishes under osteogenic conditions with human umbilical vein endothelial cells (HUVECs) to obtain homotypic or heterotypic cell sheets (CSs). Cells were retrieved as sheets from the dishes after incubation at $20^{\circ} \mathrm{C}$. Monoculture osteogenic CSs were stacked on top of homotypic or heterotypic CSs, and subcutaneously implanted in the dorsal flap of nude mice for 7 days. The implants showed mineralized tissue formation under both conditions. Transplanted osteogenic cells were found at the new tissue site, demonstrating CS bone-inductive effect. Perfused vessels, positive for human CD31, confirmed the contribution of HUVECs for the neovascularization of coculture CS constructs. Furthermore, calcium quantification and expression of osteocalcin and osterix genes were higher for the CS constructs, with HUVECs demonstrating the more robust osteogenic potential of these constructs. This work demonstrates the potential of using endothelial cells, combined with osteogenic CSs, to increase the in vivo vascularization of CS-based 3D constructs for bone tissue engineering purposes.

Laboratory Investigation (2014) 94, 663-673; doi:10.1038/labinvest.2014.55; published online 7 April 2014

KEYWORDS: bone tissue engineering; cell sheets; endothelial cells; osteoblasts; vascularization

The use of scaffolds in combination with osteogenic cells has been the gold standard in bone tissue engineering strategies. ${ }^{1,2}$ Engineered constructs are transplanted to patients to restore or improve the functions of bone tissue. However, such constructs have often failed to produce the desired results because of issues such as the poor biocompatibility and immunogenicity of the biomaterials used, and cell necrosis at the bulk of the scaffold related to deficient oxygen and nutrient diffusion. ${ }^{3-7}$ Oxygen and nutrient supply is a critical issue when creating thick-engineered tissues such as the bone. ${ }^{1,2}$ The consequence of this problem is that successful production of tissue-engineered products is virtually limited to thin tissues such as the skin. ${ }^{7}$ This scenario illustrates how vascularization is a major hurdle of bone tissue engineering. An approach that has been increasingly studied to overcome this issue is the in vitro prevascularization of the constructs. ${ }^{4-7}$ This strategy relies on combining with the engineered construct, endothelial cells that will self- organize in prevascular structures, and then anastomose with the host vasculature after implantation. In fact, the success of this approach to solve the vascularization issue of tissueengineered constructs strongly depends on a fast and functional anastomosis of the prevascular network with the host's vasculature. $^{7}$

CS engineering, as proposed by our group, ${ }^{10-12}$ is based on the use of thermoresponsive poly( $N$-isopropylacrylamide) (PIPPAam)-grafted culture surfaces that allow the recovery of confluent cells in the form of sheets. The principle of the method is based on the hydrophilic/hydrophobic nature of PIPPAam, below/above its low critical solution temperature, respectively, forming cell-adhesive and -repellent ${ }^{10-12}$ surfaces. Cell adhesion is therefore controllable by modulating the temperature. Using this strategy, our group has been proposing solutions for the regeneration of cornea, ${ }^{10}$ myocardium, ${ }^{13}$ periodontal ligament ${ }^{14}$ and bladder, ${ }^{15}$ among others. Recently, we proposed the use of osteogenic CSs produced using

${ }^{1}$ Institute of Advanced Biomedical Engineering and Science, Tokyo Women's Medical University, Tokyo, Japan; ${ }^{2}$ 3B's Research Group—Biomaterials, Biodegradables and Biomimetics, University of Minho, Headquarters of the European Institute of Excellence on Tissue Engineering and Regenerative Medicine, Taipas, Guimarães, Portugal and ${ }^{3} \mathrm{ICVS} / 3 \mathrm{Bs}$ PT Government Associate Laboratory, Braga/Guimarães, Portugal

Correspondence: Professor T Okano, PhD, Institute of Advanced Biomedical Engineering and Science, Tokyo Women's Medical University, 8-1 Kawada-cho, Shinjuku-ku, Tokyo 162-8666, Japan.

E-mail: tokano@abmes.twmu.ac.jp

Received 15 November 2013; revised 30 January 2014; accepted 6 March 2014 
thermoresponsive dishes for bone tissue engineering applications. ${ }^{16}$ In that study, using a single CS, we successfully obtained ectopic formation of vascularized bone with marrow. Osteogenic CSs obtained by mechanical retrieval with cell scrapers, alone or combined with scaffolds, were also tested for bone tissue engineering purposes, but the general outcome was an insufficient quantity of new bone tissue formed. ${ }^{17-19}$ However, Nakamura et a ${ }^{20}$ demonstrated the full regeneration of a rat critical size defect by using CSs recovered by a cell scraper. These studies show how scaffold-free, CS-based constructs can be applied for bone regeneration.

The recovery of CSs using thermoresponsive methods presents several advantages over the use of enzymatic and mechanical dissociation methods. It has been demonstrated that mechanical recovery of cells by scraping depletes ECM proteins from the resulting sheets. ${ }^{21}$ On the contrary, recovery of cells from thermoresponsive dishes preserves extracellular matrix proteins, ${ }^{11,12}$ which act as a natural glue, thus avoiding the use of sutures after transplantation. ${ }^{11,12}$ This advantage has also been explored to build threedimensional (3D) CS-based tissues, as the adhesion of the successive CSs occurs naturally. The vascularization of the 3D CS-based tissues is an essential issue as necrosis affects nonvascularized-transplanted tissues thicker than $100 \mu \mathrm{m} .{ }^{22}$ This was previously addressed by Shimizu and co-workers ${ }^{22}$ by multitransplanting rat cardiomyocyte CSs. Using this strategy, the authors were able to create 1-mm-thick myocardium with an organized vascular network. The prevascularization of CS-based 3D constructs has nevertheless been the focus of recent works. ${ }^{23-25}$ The combination of several myoblasts sheets, recovered from PIPPAam dishes, and human umbilical vein endothelial cells (HUVECs) using a CS stacking methodology resulted in the generation of a prevascularized cell-dense tissue. ${ }^{24}$ This work is a proof-of-concept on how CS engineering has the potential to produce 3D prevascularized tissues.

Considering the need to promote the formation of vascularized neobone tissue, the aim of the present work was to further improve our previously proposed CS-based strategy for bone tissue engineering. With this purpose, the potential of combining osteogenic CSs with endothelial cells to increase the vascularization of cell constructs was assessed, and the influence of endothelial cells over the amount of bone tissue formed was demonstrated. Rat osteogenic CSs were obtained using thermoresponsive dishes and were stacked with HUVECs cultured in between. The constructs were then implanted subcutaneously in the dorsal flap of nude mice and characterized in terms of involvement of the transplanted cells on both bone tissue formation and vascularization.

\section{MATERIALS AND METHODS}

\section{Thermoresponsive Culture Surfaces}

Thermoresponsive dishes (CellSeed, Tokyo, Japan) were prepared as described previously. ${ }^{26}$ Briefly, $\mathrm{N}$-isopropylacrylamide monomer in 2-propanol solution was spread onto 35-mm-diameter culture dishes (BD Biosciences, Franklin Lakes, NJ, USA). Dishes were then irradiated by electron beam, resulting in both polymerization and covalent grafting of PIPAAm onto the cell culture surfaces. PIPAAm-grafted dishes were rinsed with cold-distilled water to remove ungrafted monomer, and dried in nitrogen gas. Dishes were finally sterilized with ethylene oxide gas before experimental use.

\section{Cell Sheet Fabrication}

Bone marrow was flushed from the femurs of 4-week-old male wistar rats (Charles River, Yokohama, Japan). After careful pipetting to disaggregate any clumps, the suspension was seeded in $75 \mathrm{~cm}^{2}$ Primaria culture flasks (BD Biosciences) and cultured in basal medium ( $\alpha$-minimum essential medium with glutamax I, low glucose; Gibco-BRL LifeTechnologies, Grand Island, NY, USA), supplemented with $10 \%$ fetal bovine serum (Japan Bioserum, Japan) and $100 \mathrm{U} / \mathrm{ml}$ of penicillin-streptomycin (Sigma-Aldrich, Tokyo, Japan), at $37^{\circ} \mathrm{C}$ in a $5 \% \mathrm{CO}_{2}$ humidified atmosphere. After $24 \mathrm{~h}$ of culture, non-adherent cells were removed and the adherent cells were then maintained in culture until semiconfluence was achieved. Cell sheets (CSs) were fabricated and stacked as depicted in Figure 1. Rat bone marrow stromal cells (rBMSCs) were detached using a $0.25 \%$ trypsin-EDTA solution (Gibco-BRL LifeTechnologies) and seeded in 35-mm-diameter thermoresponsive dishes at a concentration of $2.5 \times 10^{5}$ cells per dish. Cultures were maintained for 21 days in osteogenic medium (basal medium supplemented with $10^{-8} \mathrm{M}$ dexamethasone (Dexart; Fuji Pharma, Tokyo, Japan), $50 \mu \mathrm{g} / \mathrm{ml} \mathrm{L}$-ascorbic acid phosphate (Wako Pure Chemicals, Osaka, Japan), and $10 \mathrm{mM} \beta$-glycerophosphate (Sigma-Aldrich). HUVECs were purchased from Lonza (Lonza, Japan), seeded in $75 \mathrm{~cm}^{2}$ Primaria culture flasks, and cultured in endothelial cell growth medium MV2 (ECGM; Promocell, Germany).

\section{Establishment of Cocultures}

HUVECs at passage 3 were seeded on the thermoresponsive dishes with the rBMSCs, at a density of $1 \times 10^{5}$ cells per dish, 4 days before the rBMSCs completed the 21 days of osteogenic differentiation. Cocultures were maintained in ECGM supplemented with $10^{-8} \mathrm{M}$ dexamethasone, $50 \mu \mathrm{g} / \mathrm{ml}$ L-ascorbic acid phosphate (Wako), and $10 \mathrm{mM} \beta$-glycerophosphate (Sigma-Aldrich) for 3 days until transplantation.

\section{Recovery of Cells from Thermoresponsive Dishes}

To recover the cells from the thermoresponsive dishes, culture medium was removed from the culture dishes and replaced by $1 \mathrm{ml}$ of PBS (Sigma-Aldrich). A poly(vinylidene difluoride) membrane (Immobilon-P; Millipore Corporation, Billerica, MA, USA) with a diameter of $2 \mathrm{~cm}$ was placed over the rBMSC monocultures and incubated at $20^{\circ} \mathrm{C}$ for 10 min. After this time, CSs spontaneously detached from thermoresponsive dishes. CS membrane constructs were 


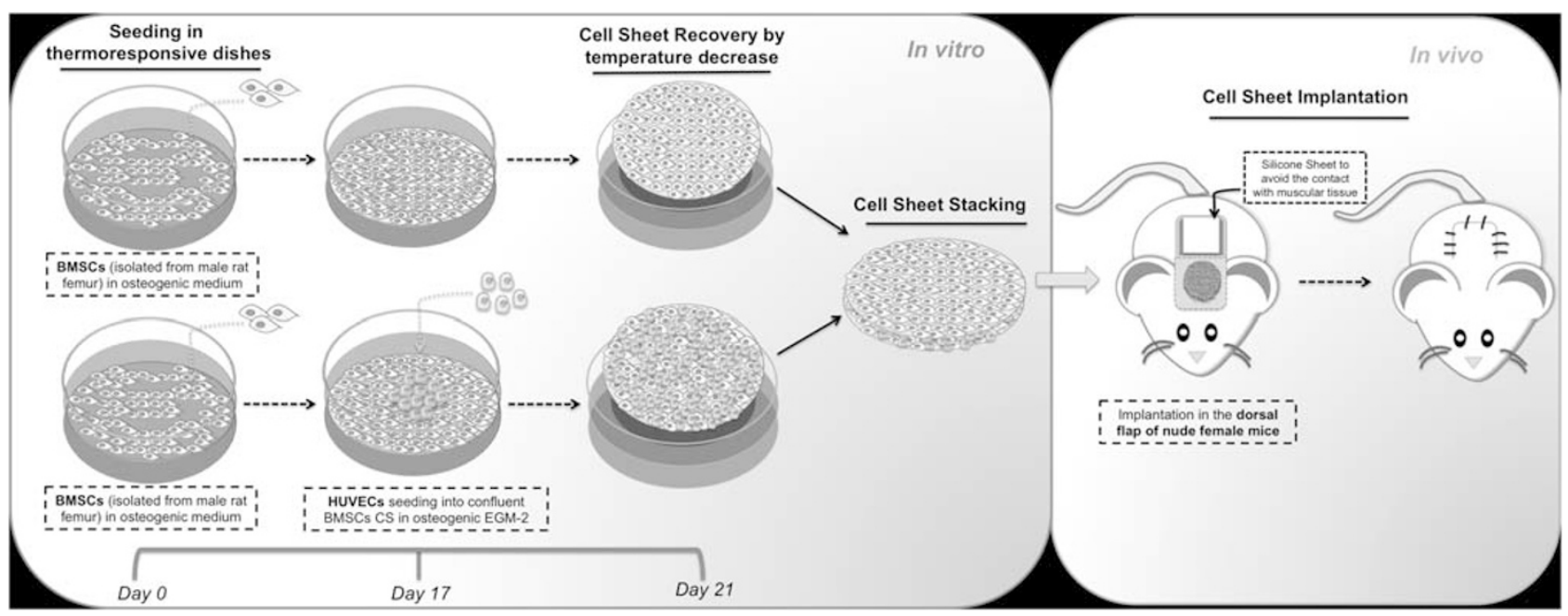

Figure 1 Subcutaneous transplantation of rat bone marrow stromal cell (rBMSCs) osteogenic cell sheets cocultured with human umbilical vein endothelial cells (HUVECs). rBMSCs were isolated from rat's femurs, expanded, and seeded in thermoresponsive dishes. After 17 days of culture in osteogenic medium, HUVECs were seeded on top of the confluent rBMSCs. Both cells were cocultured for further 4 days. CSs were retrieved from the dishes by low temperature treatment. Monocultured CSs were stacked on top of cocultured CSs and implanted on the dorsal flap of nude mice. A silicon membrane was used to cover the CSs and the flap was closed and sutured.

stacked over the rBMSC/HUVEC cocultures and the procedure to detach the adhered CSs was repeated. Controls consisted of two stacked monocultured osteogenic CS.

\section{Subcutaneous Transplantation}

Subcutaneous transplantation of the stacked CSs was carried out as reported previously. ${ }^{27}$ Briefly, 6-week-old female nude mice (Charles River) were anesthetized with a constant flux of $4 \%$ of isofluorane. Dorsal skin was cut open using $3 \times 3 \mathrm{~cm}^{2}$ cutting sides. Recovered CS constructs were placed on mouse subcutaneous dorsal flap and left to adhere to the connective tissue of dorsal skin for $5 \mathrm{~min}$. After that time, the poly(vinylidene difluoride) membranes were removed and silicone membranes were placed over the CSs to prevent the contact between the CS and the muscular tissue. Control mice were prepared by implanting constructs composed of two stacked osteogenic monocultured sheets. Skin incisions were closed using 5-0 nylon sutures. Five animals per condition, experimental and controls, were subjected to the transplantation procedure. Animals were kept with food and water ad libitum for all the experiment duration. After 7 days of transplantation, animals were killed with $\mathrm{CO}_{2}$ and implants were recovered for histological characterization.

\section{Histological Characterization}

Both in vitro recovered CS constructs and implanted samples were fixed in $4 \%$ paraformaldehyde (Wako Pure Chemicals) and embedded in paraffin, without demineralization to obtain $5-\mu$ m-thick sections. Hematoxylin and eosin (H\&E) staining was performed following standard protocols. Immunostaining was performed in both in vitro recovered CS constructs and implanted samples using the following antibodies: goat anti-mouse/-rat SRY (Santa Cruz Biotechnology, Santa Cruz, CA, USA; Yannaki et $\left.a l^{28}\right)$, rabbit anti-human/mouse/-rat collagen I (Abcam, Cambridge, MA, USA; Husse et al $^{29}$ ), rabbit anti-human/-rat/-mouse osteopontin (Abcam; Chiba et $\left.a b^{30}\right)$, rabbit anti-human/-mouse/-rat osterix (Santa Cruz Biotechnology; Strecker et al ${ }^{31}$ ) and mouse anti-human CD31 (DakoCytomation, Glostrup, Denmark; Moriyama et $a l^{32}$ ). Briefly, sections were incubated with the primary antibody overnight at $4{ }^{\circ} \mathrm{C}$ and then for $1 \mathrm{~h}$ at room temperature, with the biotinylated secondary antibody (DakoCytomation). Control samples were subjected to incubation only with the secondary antibody eg, see Supplementary Figure 1. Sections were incubated with streptavidin-HRP (DakoCytomation) solution for $20 \mathrm{~min}$ and then treated with $\mathrm{DAB}$ chromogenic substrate solution (DakoCytomation). Stained sections were analyzed with an Eclipse E800 microscope (Nikon, Tokyo, Japan). To assess the mineral deposition, alizarin red staining was performed both on the dishes with the differentiated rBMSCs and on the deparaffinized histological sections. Samples were incubated with alizarin red S solution (Sigma-Aldrich) and observed in the microscope until correct amount of color developed. Cells were then counterstained with hematoxylin and washed in ethanol and xylene.

\section{Quantitative RT-PCR Analysis} RNA extraction and CDNA production

mRNA was extracted from tissues using Trizol (Invitrogen, Carlsbad, CA, USA) reagent according to the manufacturer's instructions. Briefly, $800 \mu \mathrm{l}$ of Trizol was added per $50 \mathrm{mg}$ of 
the tissue and samples were homogenized by vigorous pipetting and maceration. Following $5 \mathrm{~min}$ incubation, $160 \mu \mathrm{l}$ of chloroform (Sigma, USA) was added to each sample; samples were then incubated for $15 \mathrm{~min}$ at $4{ }^{\circ} \mathrm{C}$ and centrifuged at 13000 r.p.m. for $15 \mathrm{~min}$ at $4{ }^{\circ} \mathrm{C}$. After the centrifugation, the aqueous part of each sample was collected and an equal volume of isopropanol (Sigma) was added. Following an overnight incubation at $-20{ }^{\circ} \mathrm{C}$, samples were centrifuged at 9000 r.p.m. for $10 \mathrm{~min}$ at $4^{\circ} \mathrm{C}$. Supernatants were discarded and pellets were washed in ethanol, centrifuged at 9000 r.p.m. for $5 \mathrm{~min}$ at $4{ }^{\circ} \mathrm{C}$, and resuspended in $12 \mu$ l of RNase/DNase free water (Gibco, Paisley, UK). RNA quantity and purity were assessed with a NanoDrop ND-1000 spectrophotometer (NanoDrop Technologies, Wilmington, DE, USA). Samples with a 260/280 ratio between 1.6 and 2.0 were used for cDNA synthesis. The cDNA synthesis was performed using iScript cDNA synthesis kit (Bio-Rad, USA) and the MiniOpticon Real-Time PCR Detection System (BioRad). An initial amount of $2 \mu \mathrm{g}$ of mRNA was used in a total volume of $20 \mu \mathrm{l}$ of RNase/DNase free water.

\section{Quantitative real-time $P C R$}

Mouse OCN (osteocalcin), COL1a1 (collagen I), and OSX (osterix) transcripts were quantified in the cDNA samples using a quantitative real-time PCR reaction. For each sample, $G A P D H$ was used as the housekeeping gene. The primers were designed using the Primer 3 software (v. 0.4.0), inserted in PrimerBLAST to check for specificity, and synthesized by MWG Biotech (Germany) as follows: OCN forward, $5^{\prime}$-CT GAGTCTGACAAAGCCTTC- $3^{\prime}$ and $O C N$ reverse, $5^{\prime}$-GCTG TGACATCCATACTTGC-3'; COL1A1 forward, 5'-GAGCGG AGAGTACTGGATCG- $3^{\prime}$ and COL1A1 reverse, 5'-GCTTCT TTTCCTTGGGGTTC-3'; OSX forward, 5'-GCTCCTCGGTT CTCTCCATCTG- $3^{\prime}$ and OSX reverse, $5^{\prime}$-TGTGTTGCCTGG ACCTGGTG-3'; mouse GAPDH forward, 5'-ACGGCCGC ATCTTCTTGTGCA- $3^{\prime}$ and mouse GAPDH reverse, $5^{\prime}$-AAT GGCAGCCCTGGTGACCA-3'. A concentration of $200 \mathrm{nM}$ was used for all the primers in a final volume of $25 \mu \mathrm{l}$ of sample. The real-time PCR reaction was carried out using the EvaGreen SuperMix (Bio-Rad) following the manufacturer's instructions in a MiniOpticon Real-Time PCR Detection System (Bio-Rad). The relative quantification of the referred gene transcripts expression was performed using the $2^{-\Delta \Delta C T}$ method (Perkin-Elmer User Bulletin No. 2). All values were first normalized against GAPDH values and then the CSs with HUVEC values against the CS-alone values.

\section{Calcium Quantification}

Halves of the recovered implants were weighted and incubated in $0.5 \mathrm{M} \mathrm{HCl}$ to remove and dissolve the calcium. Calcium quantification in the obtained solutions was performed with the o-cresolphtalein-complexon method using the Roche Cobas kit (Roche Diagnostics, Germany) following the manufacturer's instructions. The absorbance of the samples was read at $570 \mathrm{~nm}$ in a microplate reader Synergy-HT
(Bio-Tek, Hamburg, Germany). The calcium concentrations were extrapolated from the calibration curve obtained using serial dilutions of a calcium chloride solution and then normalized with the initial tissue mass. The results for the animals belonging to each transplantation group (CS with HUVECs and CS alone) were averaged and analyzed.

\section{Statistical Analysis}

The results of the quantitative real-time PCR and calcium quantification were subjected to statistical analysis. Values for the animals of each group were averaged and analyzed using the Student's $t$-test. Results were considered significant for $P<0.05$.

\section{RESULTS \\ In vitro CS Characterization}

Osteogenic differentiation of rBMSCs in the thermoresponsive dishes was confirmed by the intense and uniform alizarin red staining for calcium deposition, observed in the culture in the osteogenic medium (Figure 2a, left dish) in contrast with the absence of staining detected in the basal medium culture (Figure 2a, right dish). The presence of HUVECs in coculture with the rBMSCs was assessed by immunohistochemistry against human CD31 (Figures $2 \mathrm{c}$ and d). HUVECs seeded on the rBMSCs cultured under osteogenic conditions, after 3 days, were organized in round or elongated colonies on top of the rBMSCs. After recovery from the thermoresponsive dishes following low temperature treatment, the organization of the HUVECs remained intact as observed after immunostaining for human CD31 in a transversal section of the cocultured CSs (Figure 3a).

After recovery from dishes following low temperature treatment, homotypic and heterotypic CSs were characterized by histology. H\&E staining revealed that the CSs were composed of a thick collagenous matrix (Figure 3b). Alizarin red staining (Figure 3c) confirmed matrix mineralization in the sheets. The osteogenic character of the CSs was further corroborated by the positive immunostaining for the matrix protein osteopontin (Figure 3d) and for the transcription factor osterix (Figure 3e). The male origin of the cells forming the sheets was also confirmed by the positivity for SRY marker (Figure 3f).

\section{Implants' Characterization}

The characterization of the implanted coculture and control constructs was performed 7 days after implantation. The formation of the new tissue was assessed by H\&E staining (representative images in Figures $4 \mathrm{a}$ and $\mathrm{b}$ ). The new tissue developed in patches along the dorsal flap of the mice; the patches are apparently larger in the cocultured (Figure 4a) than in the control (Figure $4 \mathrm{~b}$ ) conditions. The same trend was visible when comparing the different mice transplanted with the different types of CSs indicating the reproducibility of our procedures. The deposition and mineralization of an osteogenic matrix was confirmed by the presence of the bone 


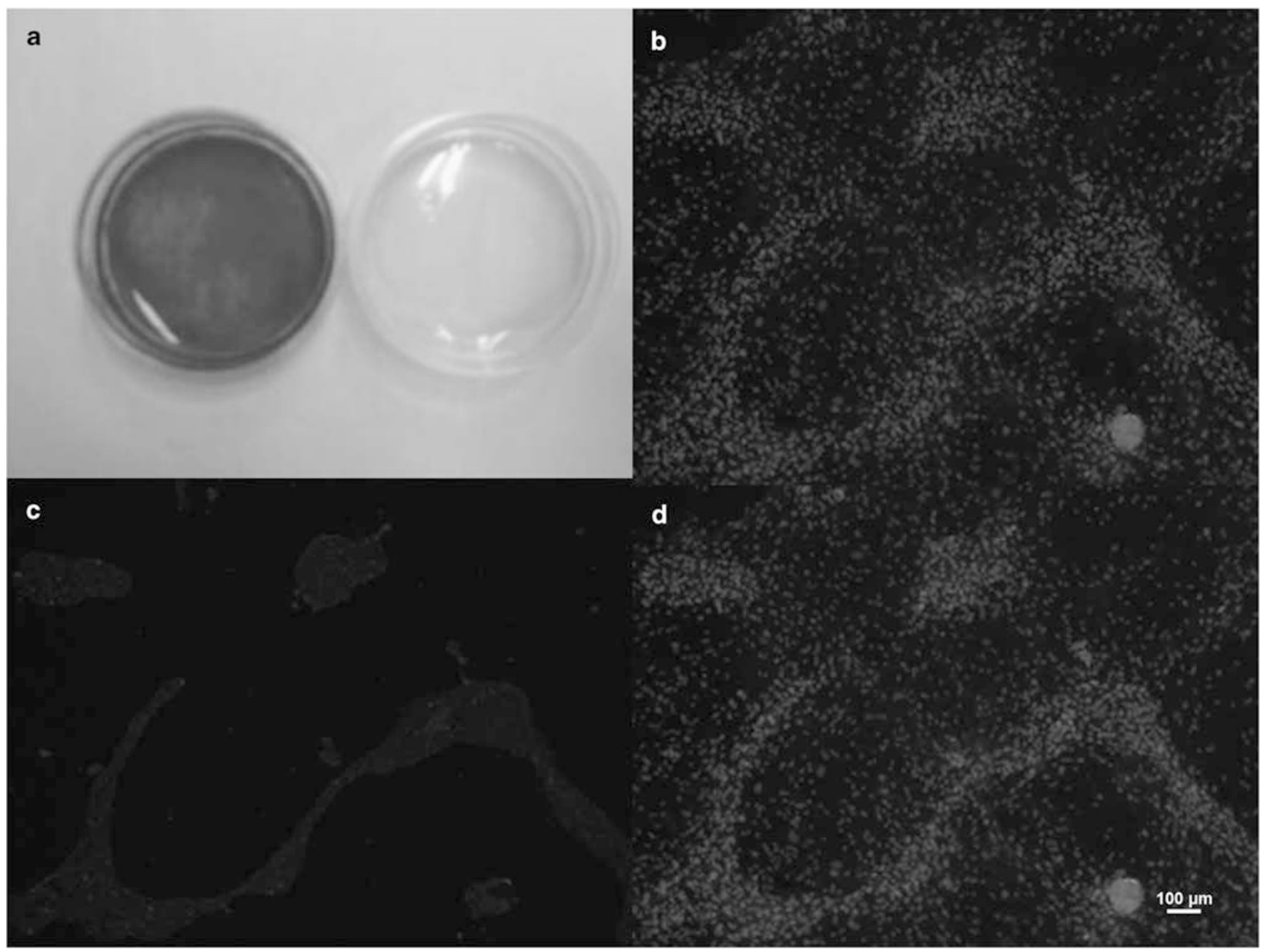

Figure 2 Alizarin red staining (a) and human CD31 immunostaining (c, d) of osteogenic cell sheets cocultured with human umbilical vein endothelial cells (HUVECs) just before retrieval from thermoresponsive dishes. Rat bone marrow stromal cells (rBMSCs) after 21 days of osteogenic differentiation (a, left side dish) and cultured in basal medium (a, right side dish). The nuclei of all cells are stained blue with 4',6-diamidino-2-phenylindole (DAPI) nuclear staining (b) and HUVECs (human CD31-positive) distribution is depicted in green. A merge micrograph of (b) and (c) is presented in (d).

protein osteopontin (Figures $4 \mathrm{c}$ and $\mathrm{d}$ ) surrounding the mineralized tissue, as determined by alizarin red staining (Figures $4 \mathrm{e}$ and $\mathrm{f}$ ). Comparing the SRY and the osterix stainings that, respectively, identify only rat male cells (Figures $5 \mathrm{a}$ and $\mathrm{b}$ ) and both mouse and rat cells (Figures $5 \mathrm{c}$ and d), it was possible to conclude that osteogenic cells both from the transplanted CSs and from the host are present and contribute to the formation of mineralized tissue. Furthermore, transcripts of bone-related genes were quantified for the retrieved implants (Figure 5e). OCN and OSX were significantly upregulated, respectively, 19.6 and 8.7 times after the implantation of the cocultured constructs and in relation to the construct without HUVECs. No statistical significant difference was detected for the COL1a1 transcripts quantity between coculture constructs and controls.

The amount of calcified tissue that was produced after the transplantation of the cocultured and control constructs was determined through calcium quantification (Figure 5f). The calcium values per amount of retrieved tissue detected for the coculture constructs were significantly higher than for the controls.

The presence of human endothelial cells in the retrieved cocultured implants was confirmed by immunostaining for human CD31 (Figure 6). Chimeric perfused vessels, formed by endothelial cells positive for human CD31, and host endothelial cells, negative for that marker, were identified in all the animals transplanted with the CSs cocultured with HUVECs.

\section{DISCUSSION}

Recently, we reported the development of osteogenic CSs using thermoresponsive dishes. ${ }^{16}$ A single osteogenic CS was able to induce the formation of vascularized new bone tissue, with marrow, after 6 weeks of implantation in nude mice. 


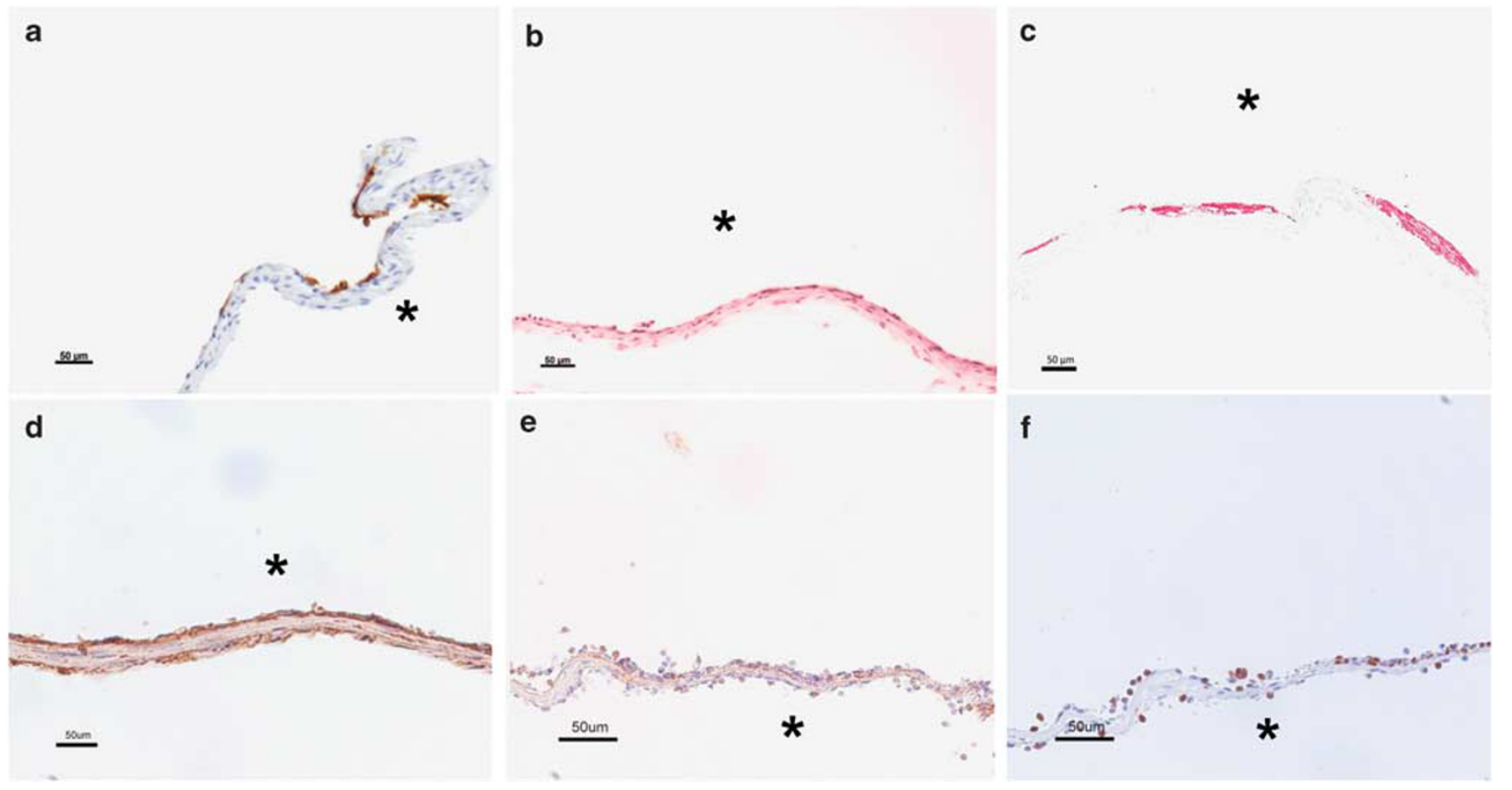

Figure 3 Histological characterization of osteogenic cell sheets after retrieval from thermoresponsive dishes. Cell sheets were stained with hematoxylin and eosin (H\&E) (b) and alizarin red (c) and immunostained for human CD31 (a) osteopontin (d), osterix (e), and SRY (f). Asterisks $(*)$ indicate basal side of the cell sheets.

Although it might not be sufficiently robust, the use of osteogenic CSs represents a valuable approach not only for bone replacement but also for bone regeneration. ${ }^{16,17,20}$ In this sense, we intended to move forward to the creation of a CS-based 3D construct and speculated that adding endothelial cells within the stacked osteogenic CSs would increase the amount of new tissue formed by promoting in vivo vascularization of the construct.

Monocultured and cocultured with HUVECs, rat osteogenic CSs were produced using thermoresponsive surfaces and the CS technology. As previously reported, ${ }^{16}$ mineralized CSs, positive for matrix protein osteopontin and for bonerelated transcription factor osterix, all strong indicators of its osteogenic nature, and composed of a thick collagenous matrix, were obtained by culturing rBMSCs in osteogenic conditions for 21 days.

The perfusion of tissue engineered constructs after transplantation is one of the most critical aspects of a successful regenerative approach. ${ }^{4,7-9}$ Strategies for improving the vascularization of tissue engineered constructs consist on the use of growth factors or on the constructs in vitro or in vivo prevascularization. ${ }^{7,33}$ The use of angiogenic growth factors is proven to increase the vascularization of implanted constructs, but the difficulty in controlling their delivery often results in disorganized and leaky vessels. ${ }^{7,33}$ In the case of in vivo prevascularization, the need to perform multiple surgeries is a serious drawback of the approach. ${ }^{7}$ For these reasons, most of the current research is focusing on ways to prevascularize constructs in vitro, using endothelial cells to accelerate its perfusion after transplantation., ${ }^{4-9}$ The potential of this strategy was demonstrated recently in a work by Takebe and co-workers. ${ }^{34}$ In that work, researchers used HUVECs and mesenchymal stem cells in coculture with hepatocytes derived from induced pluripotent stem cells, to recapitulate liver organogenesis and create an instructive niche for hepatic cells. The result was the creation in vitro of 3D prevascularized liver buds, the vessels of which were shown to connect rapidly with host vessels after implantation and to be functional in vivo. Similar efforts have been intense in the CS engineering field. ${ }^{23,24,35}$ Sasagawa et al ${ }^{24}$ seeded HUVECs between myoblast CSs, building five-layer constructs, to obtain in vitro a capillary-like network that anastomosed when implanted in nude rats, allowing the survival of the construct. Another work ${ }^{23}$ showed that the positioning of endothelial cells within two fibroblast CS constructs was determinant for tubular formation and influences its lumen area in vitro. Thus, it is increasingly clear that stacking several CS results in a suitable 3D environment for endothelial cells to form a prevascular network in vitro. In the herein presented work, it was shown that HUVECs, seeded over rBMSCs 4 days before the end of the osteogenic differentiation period, self-organized either in small round colonies or in elongated aggregates resembling a network. As these cocultured CSs were not cultured in vitro after stacking with a second osteogenic CSs, which could provide a 3D matrix for HUVECs reorganization, the 
a

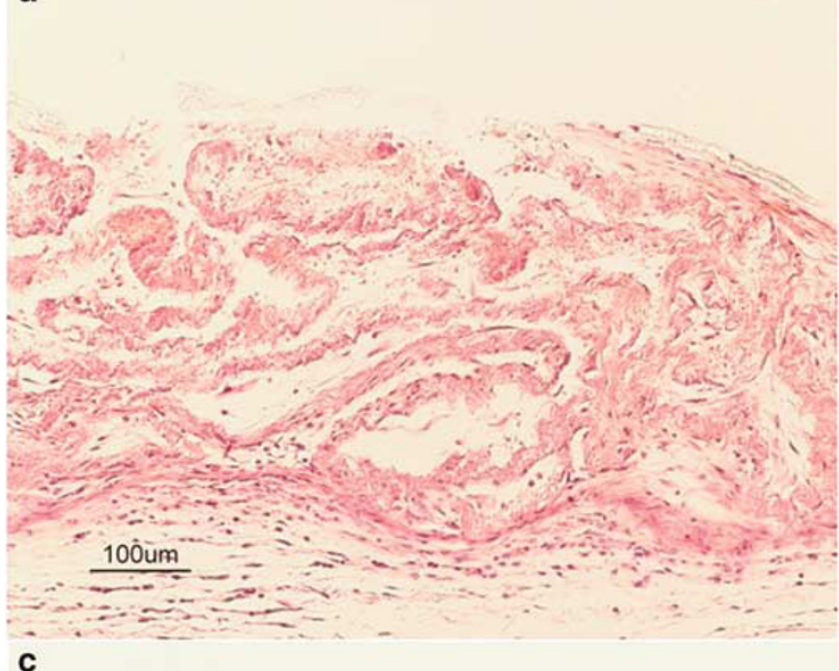

b

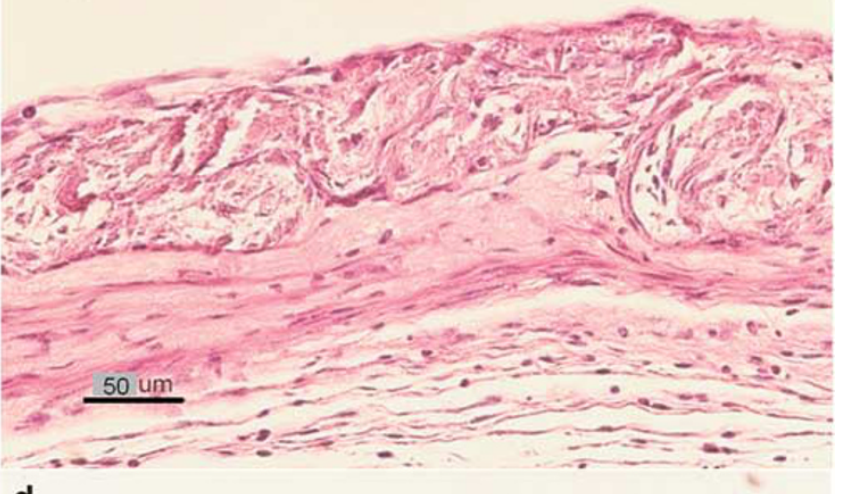

d
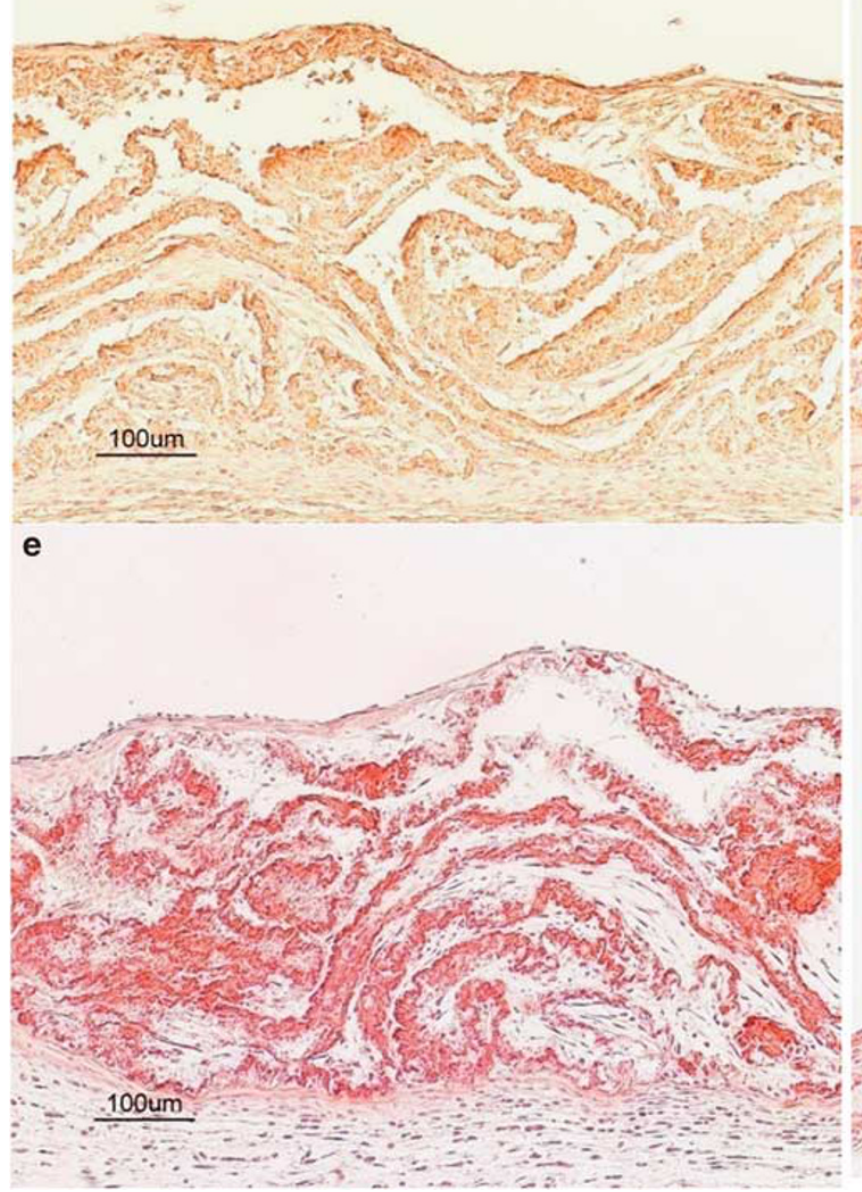
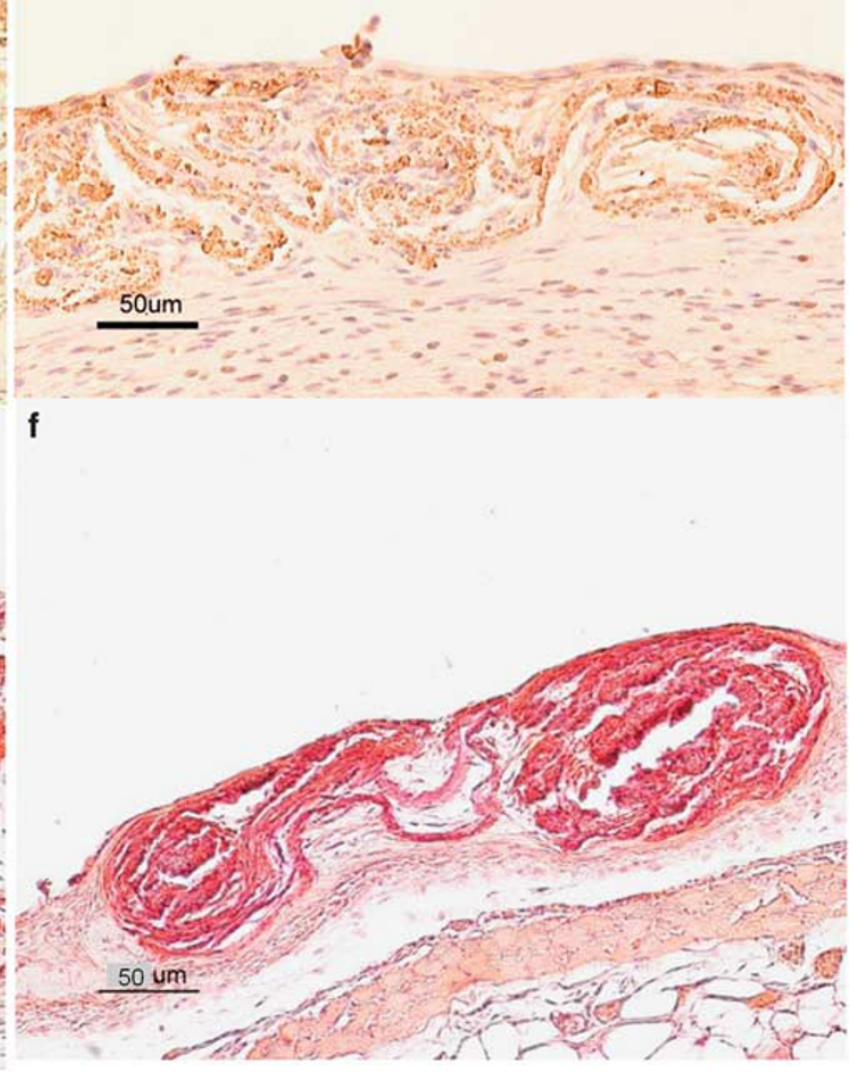

Figure 4 Histological characterization of retrieved implants 7 days after transplantation of the coculture (a, c, e) or monoculture (b, d, f) constructs. Retrieved constructs were stained with hematoxylin and eosin (H\&E) $(\mathbf{a}, \mathbf{b})$ and alizarin red $(\mathbf{e}, \mathbf{f})$ and immunostained for osteopontin $(\mathbf{c}$, $\mathbf{d})$. 


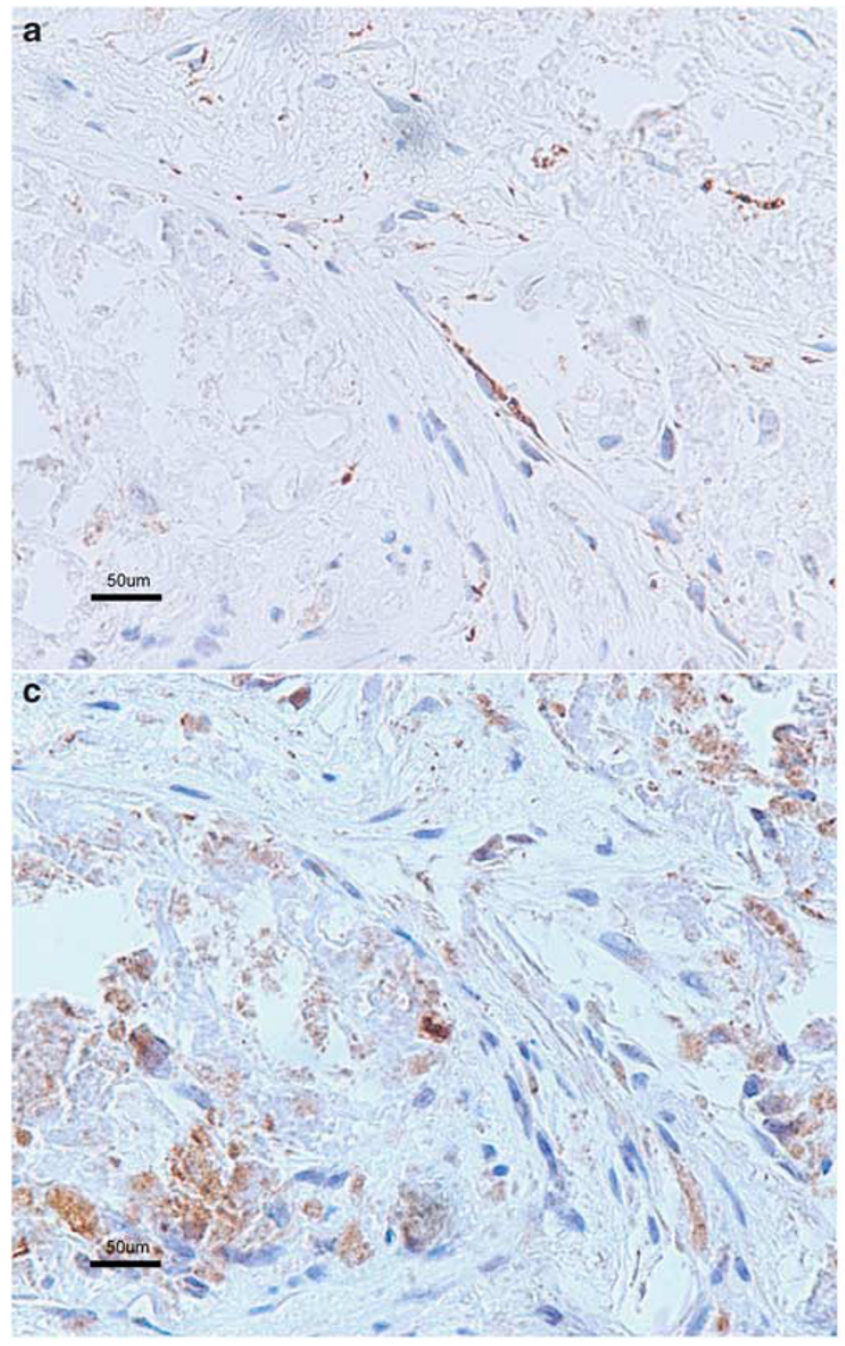

\section{b}

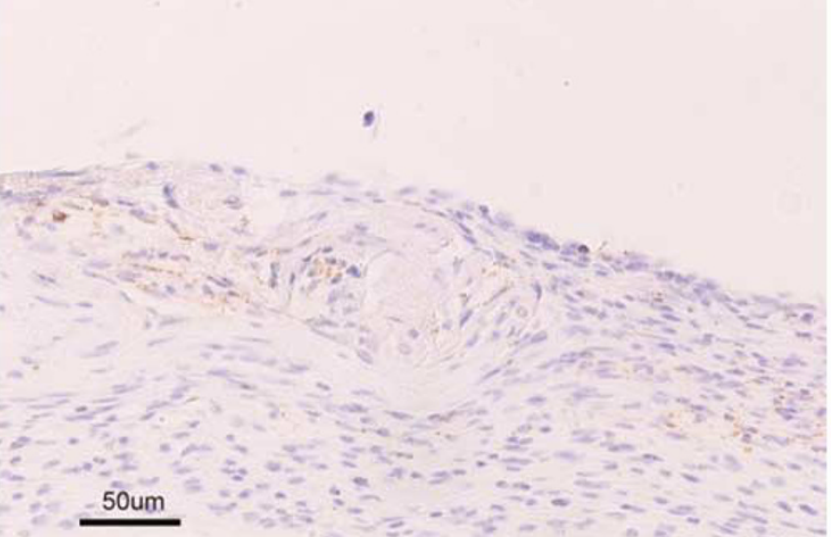

d

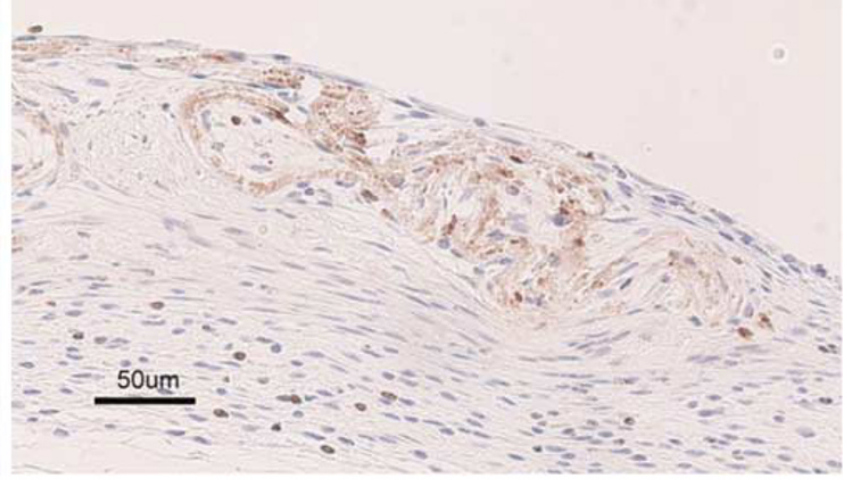

e

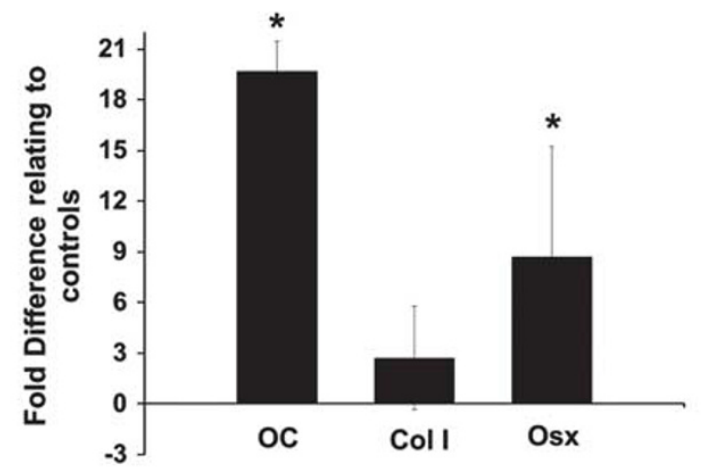

f

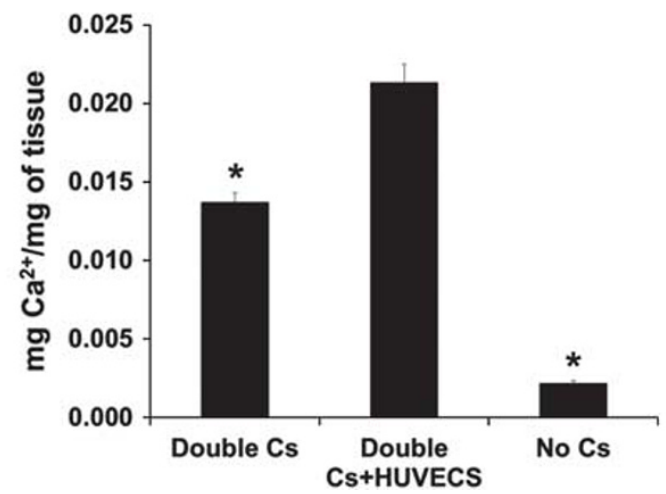

Figure 5 Immunohistological characterization of retrieved implants after implantation of the cocultured CS (a, c) or monoculture CS (b, d) constructs. Retrieved constructs were immunostained for $\operatorname{SRY}(\mathbf{a}, \mathbf{b})$ and osterix $(\mathbf{c}, \mathbf{d})$. Quantitative RT-PCR analysis (e) and calcium quantification $(\mathbf{f})$ were performed in retrieved constructs, 1 week after implantation. In (e), osteocalcin (OC), osterix (Osx) and collagen I (Col I) transcripts were quantified in both the cocultured CS and control cell sheet constructs $\left({ }^{*} P<0.05\right.$ relating to control values). Calcium was quantified using the o-cresolphtaleincomplexon method and values were normalized with tissue mass $\left({ }^{*} P<0.05\right.$ relating to coculture cell sheets).

formation of capillary-like structures, as seen in other works, ${ }^{23,24,35}$ was not expected.

The transplantation of stacked CS constructs to nude mice resulted in mineralized tissue formation after 7 days, both in the presence and absence of HUVECs. The new tissue was strongly positive for alizarin red and for the bone matrix protein osteopontin, which confirmed its osteogenic nature. Moreover, it was organized in patches throughout the flap, in 


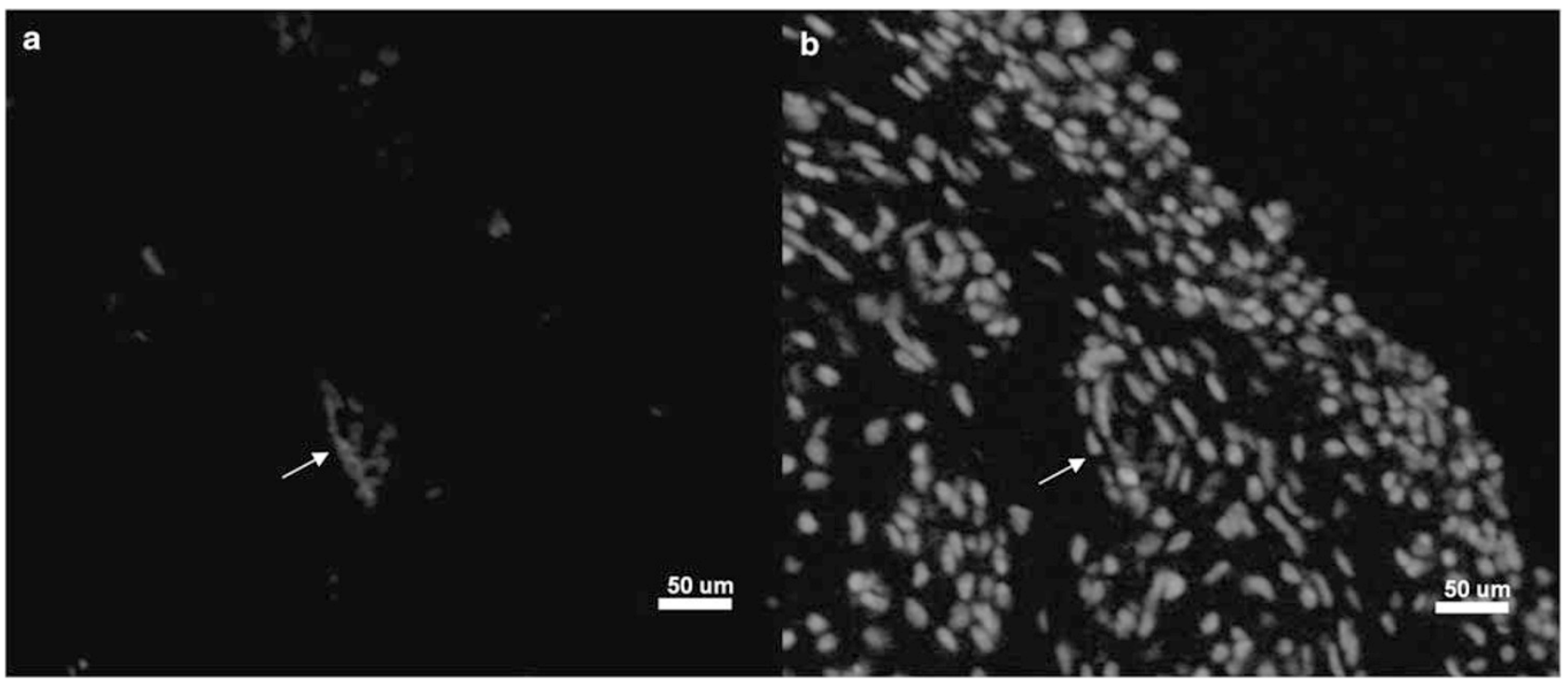

Figure 6 Immunohistological characterization of retrieved implants after implantation of the cocultured cell sheet constructs. Implants were immunostained for human CD31 (a, b) and counterstained with 4',6-diamidino-2-phenylindole (DAPI) (b). White arrows indicate perfused blood vessel.

accordance to what was observed at later time points of implantation of a single CS. ${ }^{16}$

The capacity of an implanted construct to induce the host to produce new tissue is critical in a tissue engineering strategy. The contribution to new tissue formation by the transplanted cells was discriminated from the contribution by host's cells by immunostaining for SRY. The SRY protein is encoded by a gene located in the short arm of the Y chromosome $^{36}$ and therefore can be used to identify cells from male origin and, in this case, to distinguish transplanted cells from cells from the host female mice. Therefore, the comparison of the SRY and osterix staining, the latter identifying both mouse and rat cells, permitted to verify the presence of host's osteogenic cells in addition to the transplanted ones, within the new formed tissue. This proves that the implanted CS constructs are inducing new bone formation through the recruitment of host's bone-forming cells.

Although in vitro prevascularization ensures a faster perfusion of the construct after implantation, one of its pitfalls comprehends the complex and slow determination of the ideal in vitro culture conditions and construct 3D design that allow endothelial cells to form networks, while maintaining at the same time the function of tissue-specific cells. ${ }^{4,7}$ As CSs are easy to obtain and to manipulate to assemble 3D constructs, therefore allowing a fast optimization of protocols for prevascularization, they present advantages. Furthermore, in the present work, we intended to take advantage of the mutual beneficial effects of the crosstalk between endothelial cells and osteoblasts, ${ }^{4}$ and of the intimate relation between blood vessels and bone formation ${ }^{4,37}$ to prove that the mere presence of HUVECs within the CS-based construct would be advantageous for new tissue formation. For this reason, the
CSs were only stacked right before implantation, alternatively to the pre in vitro culture of the $3 \mathrm{D}$ constructs. To verify this, we screened for the presence of HUVECs in new vessels, and quantified the mineralized tissue formed after implantation of HUVEC-containing CS-based constructs. In fact, the transplanted human endothelial cells were distributed in the new tissue, forming vessels that were perfused with erythrocytes, thus showing HUVECs' contribution to new tissue vascularization. Furthermore, calcium quantification permitted to conclude that a higher amount of mineralized tissue was formed in the coculture constructs. Based on these results, we are able to confirm that the HUVECs positively contributed to the osteogenic potential of the developed 3D CS-based constructs. This conclusion was further reinforced by quantitative RT-PCR analysis of the transcripts of the bone related genes OCN, OSX and COL1a1. After 21 days of in vitro culture in the osteogenic medium, it was expected that the totality of rBMSCs would be expressing those markers. Therefore, we wanted to verify if that expression was enhanced after coculture with HUVECs to correlate that with the amount of bone-like tissue formed. The expression of OCN and OSX was significantly increased for cocultured CS implants, which is also consistent with the higher amount of mineralized tissue formed. The similarity between conditions in the COL1a1 transcript quantification can be explained by the fact that this gene is also expressed in tissues other than bone, such as the connective tissue found in the analyzed dorsal flaps. ${ }^{38,39}$

Although it was demonstrated that the transplanted HUVECs participate in the neovascularization of the construct, we might speculate that in addition to a faster perfusion of the implant, the osteogenic effect of the endothelial 
cells ${ }^{4,37}$ also led to a higher amount of bone tissue formed. Future studies will clarify on the exact role of endothelial cells on the formation of the new mineralized tissue.

Here we demonstrate that when combined with osteogenic CS, endothelial cells participate in the neovascularization of the new tissue formed in an ectopic implantation model. More importantly, double osteogenic CS constructs, with endothelial cells in between, led to a faster and more robust formation of bone tissue, when compared with control constructs without HUVECs. This, together with the presence of HUVECs in new vessels, confirmed the positive contribution of these endothelial cells to the osteogenic potential of the developed 3D CS-based constructs. It was also demonstrated that the transplanted CS-based constructs induce bone formation through the recruitment of host's bone-forming cells. This work highlights the versatility of CS engineering for tissue engineering applications, in particular for bone tissue.

Supplementary Information accompanies the paper on the Laboratory Investigation website (http://www.laboratoryinvestigation.org)

\section{ACKNOWLEDGMENTS}

We would like to acknowledge Mariana T Cerqueira for the illustration in Figure 1. This study was supported by Formation of Innovation Center for Fusion of Advanced Technologies in the Special Coordination Funds for Promoting Science and Technology 'Cell Sheet Tissue Engineering Center (CSTEC)' and the Global COE program, the Multidisciplinary Education and Research Center for Regenerative Medicine (MERCREM), from the Ministry of Education, Culture, Sports, Science and Technology (MEXT), Japan. Financial support to RP Pirraco by the Portuguese Foundation for Science and Technology (FCT) through the PhD Grant SFRH/BD/44893/2008 is also acknowledged.

\section{DISCLOSURE/CONFLICT OF INTEREST}

The authors declare no conflict of interest. TO is a director of the board of Cell Seed, a stake holder of Cell Seed, and an inventor of cell sheet-related patent. MY had been a science consultant of Cell Seed until 2011, stake holder and an inventor of cell sheet-related patent.

1. Nerem RM, Sambanis A. Tissue engineering: from biology to biological substitutes. Tissue Eng 1995;1:3-13.

2. Salgado AJ, Coutinho OP, Reis RL. Bone tissue engineering: state of the art and future trends. Macromol Biosci 2004;4:743-765.

3. Folkman J, Hochberg M. Self-regulation of growth in three dimensions. J Exp Med 1973;138:745-753.

4. Pirraco RP, Marques AP, Reis RL. Cell interactions in bone tissue engineering. J Cell Mol Med 2009;14:93-102.

5. Holy CE, Shoichet MS, Davies JE. Engineering three-dimensional bone tissue in vitro using biodegradable scaffolds: investigating initial cell-seeding density and culture period. J Biomed Mater Res 2000; 51:376-382.

6. Ishaug-Riley SL, Crane-Kruger GM, Yaszemski MJ, et al. Threedimensional culture of rat calvarial osteoblasts in porous biodegradable polymers. Biomaterials 1998;19:1405-1412.

7. Rouwkema J, Rivron N, van Blitterswijk C. Vascularization in tissue engineering. Trends Biotechnol 2008;26:434-441.

8. Santos Ml, Tuzlakoglu K, Fuchs S, et al. Endothelial cell colonization and angiogenic potential of combined nano- and micro-fibrous scaffolds for bone tissue engineering. Biomaterials 2008;29:4306-4313.

9. Santos MI, Unger RE, Sousa RA, et al. Crosstalk between osteoblasts and endothelial cells co-cultured on a polycaprolactone-starch scaffold and the in vitro development of vascularization. Biomaterials 2009;30:4407-4415.

10. Nishida $\mathrm{K}$, Yamato $\mathrm{M}$, Hayashida $\mathrm{Y}$, et al. Corneal reconstruction with tissue-engineered cell sheets composed of autologous oral mucosal epithelium. N Engl J Med 2004;351:1187-1196.

11. Yamato M, Okano T. Cell sheet engineering. Mater Today 2004;7:42-47.

12. Yang J, Yamato $M$, Kohno $C$, et al. Cell sheet engineering: recreating tissues without biodegradable scaffolds. Biomaterials 2005;26:6415-6422.

13. Shimizu $T$, Yamato $M$, Kikuchi $A$, et al. Cell sheet engineering for myocardial tissue reconstruction. Biomaterials 2003;24:2309-2316.

14. Hasegawa M, Yamato M, Kikuchi A, et al. Human periodontal ligament cell sheets can regenerate periodontal ligament tissue in an athymic rat model. Tissue Eng 2005;11:469-478.

15. Shiroyanagi $Y$, Yamato $M$, Yamazaki $Y$, et al. Transplantable urothelial cell sheets harvested noninvasively from temperature-responsive culture surfaces by reducing temperature. Tissue Eng 2003;9:1005-1012.

16. Pirraco RP, Obokata $\mathrm{H}$, Iwata $\mathrm{T}$, et al. Development of osteogenic cell sheets for bone tissue engineering applications. Tissue Eng Part A 2011;17:1507-1515.

17. Akahane $M$, Nakamura $A$, Ohgushi $H$, et al. Osteogenic matrix sheetcell transplantation using osteoblastic cell sheet resulted in bone formation without scaffold at an ectopic site. J Tissue Eng Reg Med 2008;2:196-201.

18. Gao Z, Chen F, Zhang J, et al. Vitalisation of tubular coral scaffolds with cell sheets for regeneration of long bones: a preliminary study in nude mice. Br J Oral Maxillofac Surg 2009;47:116-122.

19. Zhou Y, Chen F, Ho ST, et al. Combined marrow stromal cell-sheet techniques and high-strength biodegradable composite scaffolds for engineered functional bone grafts. Biomaterials 2007;28:814-824.

20. Nakamura A, Akahane M, Shigematsu $H$, et al. Cell sheet transplantation of cultured mesenchymal stem cells enhances bone formation in a rat nonunion model. Bone 2010;46:418-424.

21. Canavan $\mathrm{H}$, Cheng $\mathrm{X}$, Graham D, et al. Cell sheet detachment affects the extracellular matrix: a surface science study comparing thermal liftoff, enzymatic, and mechanical methods. J Biomed Mater Res Part A 2005;75:1-13.

22. Shimizu T, Sekine H, Yang J, et al. Polysurgery of cell sheet grafts overcomes diffusion limits to produce thick, vascularized myocardial tissues. FASEB J 2006;206:708-710.

23. Asakawa N, Shimizu T, Tsuda $\mathrm{Y}$, et al. Pre-vascularization of in vitro three-dimensional tissues created by cell sheet engineering. Biomaterials 2010;31:3903-3909.

24. Sasagawa T, Shimizu T, Sekiya S, et al. Design of prevascularized threedimensional cell-dense tissues using a cell sheet stacking manipulation technology. Biomaterials 2010;31:1646-1654.

25. Sekine $H$, Shimizu $T$, Sakaguchi $K$, et al. In vitro fabrication of functional three-dimensional tissues with perfusable blood vessels. Nat Commun 2013;4:1399.

26. Hirose $\mathrm{M}$, Kwon $\mathrm{OH}$, Yamato $\mathrm{M}$, et al. Creation of designed shape cell sheets that are noninvasively harvested and moved onto another surface. Biomacromolecules 2000;1:377-381.

27. Obokata H, Yamato M, Tsuneda S, et al. Reproducible subcutaneous transplantation of cell sheets into recipient mice. Nat Protocols 2011;6:1053-1059.

28. Yannaki E, Athanasiou E, Xagorari A, et al. G-CSF-primed hematopoietic stem cells or G-CSF per se accelerate recovery and improve survival after liver injury, predominantly by promoting endogenous repair programs. Exp Hematol 2005;33:108-119.

29. Husse B, Briest W, Homagk L, et al. Cyclical mechanical stretch modulates expression of collagen I and collagen III by PKC and tyrosine kinase in cardiac fibroblasts. Am J Physiol-Regul Integr Comp Physiol 2007;293:R1898-R1907.

30. Chiba $\mathrm{H}$, Ataka $\mathrm{K}$, Iba $\mathrm{K}$, et al. Diabetes impairs the interactions between long-term hematopoietic stem cells and osteopontinpositive cells in the endosteal niche of mouse bone marrow. Am J Physiol Cell Physiol 2013;305:C693-C703.

31. Strecker S, Fu Y, Liu Y, et al. Generation and characterization of OsterixCherry reporter mice. Genesis 2013;51:246-258.

32. Moriyama M, Kumagai $\mathrm{S}$, Kawashiri $\mathrm{S}$, et al. Immunohistochemical study of tumour angiogenesis in oral squamous cell carcinoma. Oral Oncol 1997;33:369-374.

33. Jain RK, Au P, Tam J, et al. Engineering vascularized tissue. Nat Biotechnol 2005;23:821-823. 
34. Takebe T, Sekine K, Enomura M, et al. Vascularized and functiona human liver from an iPSC-derived organ bud transplant. Nature 2013;499:481-484.

35. Sekiya S, Muraoka M, Sasagawa T, et al. Three-dimensional cell-dense constructs containing endothelial cell-networks are an effective too for in vivo and in vitro vascular biology research. Microvasc Res 2010;80:549-551.

36. Wallis $M$, Waters $P$, Graves J. Sex determination in mammals-before and after the evolution of SRY. Cell Mol Life Sci 2008;65:3182-3195.
37. Collin-Osdoby P. Role of vascular endothelial cells in bone biology. J Cell Biochem 1994;55:304-309.

38. Gay S, Martin GR, Muller PK, et al. Simultaneous synthesis of types I and III collagen by fibroblasts in culture. Proc Natl Acad Sci USA 1976;73:4037-4040.

39. Gardner $\mathrm{H}$, Broberg A, Pozzi A, et al. Absence of integrin alpha1beta1 in the mouse causes loss of feedback regulation of collagen synthesis in normal and wounded dermis. J Cell Sci 1999:112(Part 3):263-272. 